\title{
A FAMILY OF 2-DIMENSIONAL MINKOWSKI PLANES WITH SMALL AUTOMORPHISM GROUPS
}

\author{
by \\ Günter F. Steinke \\ Department of Mathematics and Statistics \\ University of Canterbury, Christchurch, New Zealand
}

No. 105

March, 1994

\begin{abstract}
This paper concerns 2-dimensional (topological locally compact connected) Minkowski planes. It uses a construction of J. Jakóbowski [4] of Minkowski planes over half-ordered fields and applies it to the field of reals. This generalizes a construction by A. Schenkel [7] of 2-dimensional Minkowski planes with 3-dimensional kernel. It is shown that most planes in this family of Minkowski planes have 0-dimensional and even trivial automorphism groups.
\end{abstract}

To appear in Results of Mathematics. 


\title{
A FAMILY OF 2-DIMENSIONAL MINKOWSKI PLANES WITH SMALL AUTOMORPHISM GROUPS
}

\author{
GÜNTER F. STEINKE \\ Department of Mathematics \\ University of Canterbury \\ Christchurch, New Zealand
}

\begin{abstract}
This paper concerns 2-dimensional (topological locally compact connected) Minkowski planes. It uses a construction of J. Jakóbowski [4] of Minkowski planes over half-ordered fields and applies it to the field of reals. This generalizes a construction by $\mathrm{A}$. Schenkel [7] of 2-dimensional Minkowski planes with 3-dimensional kernel. It is shown that most planes in this family of Minkowski planes have 0-dimensional and even trivial automorphism groups.
\end{abstract}

\section{Introduction and notation}

A Minkowski plane $\mathcal{M}=\left(P, \mathcal{K},\left\{\left\|_{+},\right\|_{-}\right\}\right)$consists of a set of points $P$, a set of at least two circles $\mathcal{K}$ (considered as subsets of $P$ ) and two equivalence relations $\|_{+}$and $\|_{-}$on $P$ (parallelisms) such that three pairwise non-parallel points (that is, neither (+)-parallel nor (-)-parallel) can be joined by a unique circle, such that the circles which touch a fixed circle $K$ at $p \in K$ partition $P \backslash|p|$ (where $|p|=|p|_{+} \cup|p|_{-}$ denotes the union of the two parallel classes of $p$ ), such that each parallel class meets each circle in a unique point (parallel projection), such that each $(+)$-parallel class and each (-)-parallel class intersect in a unique point, and such that there is a circle that contains at least three points (compare [7]). A topological Minkowski plane is a Minkowski plane in which the point set $P$ and the set of circles $\mathcal{K}$ carry topologies such that the geometric operations of joining, touching, the parallel projections, intersecting parallel classes of different type, and intersecting circles are continuous operations on their domains of definition (see [7]). A topological Minkowski plane is

1991 Mathematics Subject Classification. $51 \mathrm{H} 15$.

Typeset by $\mathcal{A}_{\mathcal{M}} \mathcal{S}-\mathrm{TEX}_{\mathrm{X}}$ 
called (locally) compact, connected, or finite-dimensional if the point space has the respective topological property. According to $[5,2.3]$ a compact connected finitedimensional Minkowski plane can only be of dimension 2 or 4 . Whereas there are many models of 2-dimensional Minkowski planes (see [7], [2], [9] and [1]) no other 4-dimensional Minkowski plane than the plane over the complex numbers is known yet. In this note we consider 2-dimensional (compact connected) Minkowski planes exclusively.

The classical model of a 2-dimensional Minkowski plane is obtained as the geometry of non-trivial plane sections of a ruled quadric in the real projective 3dimensional space. In this case the topologies on the point set and the set of circles are induced from the surrounding projective 3-space.

Each circle and each parallel class of a 2-dimensional Minkowski plane is homeomorphic to the 1 -sphere $\mathbb{S}^{1}$ and the point space of such a plane homeomorphic to $\mathbb{S}^{1} \times \mathbb{S}^{1}$. The space of circles is homeomorphic to $\mathrm{PGL}(2, \mathbb{R})$ and has two connected components. Conversely, a Minkowski plane with point space homeomorphic to $\mathbb{S}^{1} \times \mathbb{S}^{1}$ is a topological 2-dimensional plane if each circle and each parallel class is homeomorphic to $\mathbb{S}^{1}$, cf. [7, Satz 4.4]. Each such Minkowski plane $\mathcal{M}$ can be represented as follows. The point set of $\mathcal{M}$ is $\mathbb{S}^{1} \times \mathbb{S}^{1}$, parallel classes are of the form $\left\{x_{0}\right\} \times \mathbb{S}^{1}$ and $\mathbb{S}^{1} \times\left\{y_{0}\right\}$ for $x_{0}, y_{0} \in \mathbb{S}^{1}$. Each circle $K$ of $\mathcal{M}$ can be described by a continuous function $f_{K}: \mathbb{S}^{1} \rightarrow \mathbb{S}^{1}$ as

$$
K=\left\{\left(x, f_{K}(x)\right) \mid x \in \mathbb{S}^{1}\right\} .
$$

The axiom of parallel projection shows that each function $f_{K}$ is a homeomorphism of $\mathbb{S}^{1}$. The axiom of joining implies that the collection of all those homeomorphisms $f_{K}$ is a sharply 3 -transitive set of permutations of $\mathbb{S}^{1}$. If we use this representation, we say that the Minkowski plane is in standard representation. Conversely, each such incidence structure constructed from a sharply 3-transitive set of permutations of $\mathbb{S}^{1}$ is equivalent to a more general hyperbola structure, that is, all axioms of a Minkowski plane are satisfied except the axiom of touching.

Associated withevery point $p$ of $\mathcal{M}$ there is an incidence structure, called the derived affine plane $\mathcal{A}_{p}=\left(A_{p}, \mathcal{L}_{p}\right)$ at $p$, whose point set $A_{p}$ consists of all points of $\mathcal{M}$ that are not parallel to $p$ and whose set of lines $\mathcal{L}_{p}$ consists of all restrictions to $A_{p}$ of circles of $\mathcal{M}$ passing through $p$ and of all parallel classes not passing through $p$. Indeed, $\mathcal{M}$ is a Minkowski plane if and only if all incidence structures $\mathcal{A}_{p}$ are affine planes.

Each automorphism of a 2-dimensional Minkowski plane is continuous. The group $\Gamma=A u t(\mathcal{M})$ of all automorphisms of $\mathcal{M}$ carries the compact-open topology. $\Gamma$ is a Lie group of dimension at most 6 in this topology; cf. [7, Sätze 5.6, 5.7] or [8]. Moreover, $\Gamma$ has two distinguished closed normal subgroups

$$
T^{ \pm}=\left\{\gamma \in \Gamma \mid x \|_{ \pm} \gamma(x) \text { for all } x \in P\right\}
$$

called the kernels. Each kernel can be at most 3-dimensional.

The classical real Minkowski plane was characterized by A. Schenkel [7, Kor. 5.9, Satz 5.10] by the following 
1.1. Theorem. A 2-dimensional Minkowski plane $\mathcal{M}$ is isomorphic to the classical real Minkowski plane if and only if any one of the following holds:

(1) one of the normal subgroups $T^{ \pm}$is 3-dimensional and at least one derived plane is Desarguesian;

(2) the automorphism group of $\mathcal{M}$ is at least 5-dimensional.

2-dimensional Minkowski planes with 4-dimensional automorphism groups were also classified by A. Schenkel [7, Satz 5.11]. For $r, s \in \mathbb{R}^{+}$let $f_{r, s}$ be the orientationpreserving homeomorphism of $\mathbb{S}^{1} \simeq \mathbb{R} \cup\{\infty\}$ defined by

$$
f_{r, s}(x)= \begin{cases}x^{r}, & \text { if } x \in \mathbb{R}, x \geq 0 \\ -s|x|^{r}, & \text { if } x \in \mathbb{R}, x<0 \\ \infty, & \text { if } x=\infty\end{cases}
$$

1.2. Theorem. A 2-dimensional Minkowski plane admitting a 4-dimensional automorphism group is isomorphic to $\mathcal{M}\left(f_{r, 1}, i d\right)$ for some $r>0$, see 2.1 below for this type of planes, or it is isomorphic to a Minkowski plane $\mathcal{M}\left(r_{1}, s_{1} ; r_{2}, s_{2}\right)$, where $r_{1}, s_{1}, r_{2}, s_{2} \in \mathbb{R}^{+}$, of the following form: the plane is in standard representation and the circle set of $\mathcal{M}\left(r_{1}, s_{1} ; r_{2}, s_{2}\right)$ consists of all Euclidean lines extended by the point $(\infty, \infty)$ and the sets $\left\{\left(x, \frac{a}{f_{r_{1}, b_{1}}(x-b)}+c\right) \mid x \in \mathbb{S}^{1}\right\}$ and $\left\{\left(x, \frac{-a}{f_{r_{2}, b_{2}}(x-b)}+c\right) \mid x \in \mathbb{S}^{1}\right\}$ for all $a \in \mathbb{R}^{+}, b, c \in \mathbb{R}$, where we use the convention $\frac{1}{\infty}=0$ and $a \cdot \infty+b=\infty$ for all $a, b \in \mathbb{R}, a \neq 0$.

The planes $\mathcal{M}\left(r_{1}, s_{1} ; r_{2}, s_{2}\right)$ generalize Hartmann's [2] construction of 2-dimensional Minkowski planes, which in the above notation are the planes $\mathcal{M}\left(r_{1}, 1 ; r_{2}, 1\right)$.

In this note we investigate a family of Minkowski planes most of which have 0 -dimensional and even trivial automorphism groups. These planes were first constructed by J. Jakóbowski in a slightly different form (and more generally over half-ordered fields, cf. [4]) and are generalization of the planes with 3-dimensional kernel obtained by A. Schenkel. Although most of the following carries over to the general setting over half-ordered fields, we restrict ourselves to the real situation.

\section{The Minkowski planes $\mathcal{M}(f, g)$}

In the remainder of this note let $\Sigma$ denote the projective linear group PGL(2, R), that is, the quotient group formed by the general linear group $G L(2, \mathbb{R})$ of real $2 \times 2$ matrices modulo the non-zero scalar matrices. Each element of $\Sigma$ can be represented by a $2 \times 2$ matrix $\left(\begin{array}{ll}a & b \\ c & d\end{array}\right), a d-b c \neq 0$, which operates on the set of 1-dimensional subspaces of $\mathbb{R}^{2}$ like the fractional linear mapping $x \mapsto \frac{a x+b}{c x+d}$ on $\mathbb{S}^{1}=\mathbb{R} \cup\{\infty\}$. We further denote the subgroup $\operatorname{PSL}(2, \mathbb{R})$ by $\Delta$. This is a normal subgroup of index 2 in $\Sigma$. In the natural operation of $\Sigma$ on $\mathbb{S}^{1}$ given above $\Delta$ consists of orientation-preserving homeomorphisms of $\mathbb{S}^{1}$ whereas $\Sigma \backslash \Delta$ consists of orientation-reversing homeomorphisms of $\mathbb{S}^{1}$. It is well known that $\Sigma$ is a sharply 3 -transitive permutation group of $\mathbb{S}^{1}$. 
2.1. The incidence structures $\mathcal{M}(f, g)$.

Let $f$ and $g$ be two orientation-preserving homeomorphisms of $\mathbb{S}^{1}$. We define

$$
\mathcal{K}_{f, g}=\Delta \cup g^{-1}(\Sigma \backslash \Delta) f .
$$

$\mathcal{M}(f, g)$ is the following incidence structure. The point set of $\mathcal{M}(f, g)$ is $\mathbb{S}^{1} \times \mathbb{S}^{1}$, parallel classes are of the form $\left\{x_{0}\right\} \times \mathbb{S}^{1}$ and $\mathbb{S}^{1} \times\left\{y_{0}\right\}$ for $x_{0}, y_{0} \in \mathbb{S}^{1}$. The set of circles of $\mathcal{M}(f, g)$ is $\mathcal{K}_{f, g}$, that is, circles are of the form

$$
\left\{(x, \gamma(x)) \mid x \in \mathbb{S}^{1}\right\}
$$

for $\gamma \in \Delta \cup g^{-1}(\Sigma \backslash \Delta) f$.

$f=g=i d$ yields the classical real Minkowski plane. When $g=i d$ each mapping $(x, y) \mapsto(x, \delta(y))$ for $\delta \in \Delta$ is an automorphism of $\mathcal{M}(f, i d)$. This shows that $\mathcal{M}(f, i d)$ has a 3-dimensional kernel $T^{+}$. Conversely, A. Schenkel proved in [7, Satz 5.9]

2.2. Theorem. A 2-dimensional Minkowski plane admitting a 3-dimensional $\mathrm{k}$ ernel is isomorphic to a plane $\mathcal{M}(f, i d)$ as in 2.1 for some orientation-preserving homeomorphism $f$ of $\mathbb{S}^{1}$.

The planes $\mathcal{M}(f, g)$ also comprise, up to isomorphisms, all semiclassical 2-dimensional Minkowski planes. These are planes whose point set is the union of two open connected subsets and certain circles and parallel classes which form the common boundary of both open sets and such that as few as possible circles and parallel classes are used. The induced topology and geometry on each open subset is isomorphic to the topology and geometry of the classical real Minkowski plane on a corresponding set. So the construction of semiclassical planes can be imagined as two halves of a classical plane being pasted together along certain circles or parallel classes. In the above notation the semiclassical 2-dimensional Minkowski planes are precisely the planes $\mathcal{M}\left(f_{1, q}, i d\right)$ for some $q>0$. They are obtained by pasting along two parallel classes of the same type.

In order to prove that $\mathcal{M}(f, g)$ is a 2 -dimensional Minkowski plane we need a wellknown lemma that allows us to recognize orientation-preserving homeomorphisms of $\mathbb{S}^{1}$ by the number of their fixed points.

2.3. Lemma. Let $\phi$ be a homeomorphism of $\mathbb{S}^{\mathbf{1}}$. Suppose that $\phi$ fixes either at least three points or precisely one point. Then $\phi$ is orientation-preserving.

Proof. Since $\Delta$ is transitve on $\mathbb{S}^{1}=\mathbb{R} \cup\{\infty\}$ and because conjugation by any homeomorphism preserves the original orientation, we may assume that $\infty$ is a fixed point of $\phi$. Then $\phi$ induces a homeomorphism $\phi^{\prime}$ of $\mathbb{R}$. Such a homeomorphism is either strictly increasing or strictly decreasing. The former case corresponds to an orientation-preserving homeomorphism of $\mathbb{S}^{1}$. In the latter situation $\phi^{\prime}$ comes from an orientation-reversing homeomorphism of $\mathbb{S}^{1}$. The assumptions made about $\phi \mathrm{im}-$ ply that $\phi^{\prime}$ fixes either at least two points or no point of $\mathbb{R}$. Obviously, in the former case $\phi^{\prime}$ must be strictly increasing, and consequently, $\phi$ is orientation-preserving. In the latter case $\phi^{\prime}$ cannot be strictly decreasing because such a homeomorphis$m$ of $\mathbb{R}$ must have a fixed point by the intermediate value theorem. Hence, $\phi$ is orientation-preserving in both cases. 
2.4. Corollary. $\mathcal{K}_{f, g}$ is a sharply 3 -transitive set of permutations of $\mathbb{S}^{1}$.

Proof. Let $x_{i}, y_{i} \in \mathbb{R}, i=1,2,3$, such that the $x_{i}$ 's and also the $y_{i}$ 's are pairwise distinct. By the 3-transitivity of $\Sigma$ on $\mathbb{S}^{1}$ we find $\sigma, \sigma^{\prime} \in \Sigma$ with $\sigma\left(x_{i}\right)=y_{i}$ and $\sigma^{\prime}\left(f\left(x_{i}\right)\right)=g\left(y_{i}\right)$ for $i=1,2,3$. Since $\sigma^{-1} g^{-1} \sigma^{\prime} f$ fixes the three points $x_{1}, x_{2}, x_{3}$, this mapping must be orientation-preserving by Lemma 2.3. Hence $\sigma$ and $g^{-1} \sigma^{\prime} f$ are either both orientation-preserving or both orientation-reversing. Since $f$ and $g$ are orientation-preserving, the orientations of $g^{-1} \sigma^{\prime} f$ and $\sigma^{\prime}$ agree. Hence, $\sigma$ and $\sigma^{\prime}$ are either both in $\Delta$ or both in $\Sigma \backslash \Delta$. This shows that there is precisely one element in $\Delta \cup g^{-1}(\Sigma \backslash \Delta) f$ that maps $x_{i}$ to $y_{i}$ for $i=1,2,3$ - namely, $\sigma$, if it is in $\Delta$, or $g^{-1} \sigma^{\prime} f$, if $\sigma \in \Sigma \backslash \Delta$.

2.5. Corollary. If two distinct circles in $\mathcal{K}_{f, g}$ described by $\gamma$ and $\gamma^{\prime}$ touch each other, then $\gamma$ and $\gamma^{\prime}$ are either both orientation-preserving or both orientationreversing.

Proof. Since the circles described by $\gamma$ and $\gamma^{\prime}$ touch each other, $\gamma^{-1} \gamma^{\prime}$ has precisely one fixed point. The conclusion then follows from Lemma 2.3.

2.6. Theorem. $\mathcal{M}(f, g)$ is a 2-dimensional Minkowski plane.

Proof. By Corollary 2.4 the incidence structure $\mathcal{M}(f, g)$ is a hyperbola structure in standard representation. To verify that $\mathcal{M}(f, g)$ is a Minkowski plane we only have to verify the axiom of touching. Given a circle $\gamma \in \mathcal{K}_{f, g}$, we know from Corollary 2.5 that a circle through $\left(x_{1}, y_{1}\right)$ that touches $\gamma$ at $\left(x_{0}, y_{0}\right)$ is necesarily described by a $\gamma^{\prime}$ of the same orientation as $\gamma$. So, if $\gamma \in \Delta$ then $\gamma^{\prime} \in \Delta$ too and $\gamma^{\prime}$ can uniquely be found in the classical real Minkowski plane. Similarly, if $\gamma \in g^{-1}(\Sigma \backslash \Delta) f$, i.e. $\gamma=g^{-1} \sigma f$ for $\sigma \in \Sigma \backslash \Delta$, then $\sigma^{\prime} \in \Sigma \backslash \Delta$ can be uniquely found in the classical real Minkowski plane as the circle through $\left(f\left(x_{1}\right), g\left(y_{1}\right)\right)$ that touches $\sigma$ at $\left(f\left(x_{0}\right), g\left(y_{0}\right)\right)$. Then $\gamma^{\prime}=g^{-1} \sigma^{\prime} f$ describes the circle through $\left(x_{1}, y_{1}\right)$ that touches $\gamma$ at $\left(x_{0}, y_{0}\right)$. This shows that there is a unique circle through $\left(x_{1}, y_{1}\right)$ that touches $\gamma$ at $\left(x_{0}, y_{0}\right)$.

Obviously, each parallel class is homeomorphic to $\mathbb{S}^{1}$. Since each circle is described as the graph of a continuous bijection of $\mathbb{S}^{1}$, each circle is also homeomorphic to $\mathbb{S}^{1}$. Hence $\mathcal{M}(f, g)$ is a (topological compact) 2-dimensional Minkowski plane by [7, Satz 4.4].

2.7. Remark. a) Substituting $f$ and $g$ by $\delta f$ and $\delta^{\prime} g$ respectively for $\delta, \delta^{\prime} \in \Delta$ does not alter the circle set, that is, $\mathcal{M}(f, g)=\mathcal{M}\left(\delta f, \delta^{\prime} g\right)$. Since $\Delta$ is still 2-transitive and because the stabilizer $\Delta_{x, y}$ of two points $x, y$ is transitive on each connected component of $\mathbb{S}^{1} \backslash\{x, y\}$, we may assume, if necessary, that $f$ and $g$ both fix $\infty, 1$ and 0 .

b) The homeomorphism $\mathbb{S}^{1} \rightarrow \mathbb{S}^{1}:(x, y) \mapsto(y, x)$ defines an isomorphism from $\mathcal{M}(f, g)$ to $\mathcal{M}(g, f)$; cf. Theorem 3.14. A circle $K_{\tau}, \tau \in \mathcal{M}_{f, g}$, is mapped to $K_{\tau^{-1}}$ and $\tau^{-1} \in \mathcal{M}_{g, f}$. 


\section{Automorphisms of $\mathcal{M}(f, g)$}

3.1. Let $\Gamma_{f, g}$ denote the automorphism group of $\mathcal{M}(f, g)$. An automorphism $\gamma \in$ $\Gamma_{f, g}$ has the form $(x, y) \mapsto(\alpha(x), \beta(y))$ or the form $(x, y) \mapsto(\alpha(y), \beta(x))$ for two homeomorphisms $\alpha, \beta$ of $\mathbb{S}^{1}$ depending on whether $\gamma$ maps $(+)$-parallel classes to $(+)$-parallel classes and (-)-parallel classes to $(-)$-parallel classes or exchanges $(+)$ and (-)-parallel classes. In the former case, $\gamma$ maps a circle $K_{\tau}, \tau \in \mathcal{K}_{f, g}$, to $K_{\beta \tau \alpha^{-1}}$. In the latter case, $K_{\tau}, \tau \in \mathcal{K}_{f, g}$, is taken to $K_{\beta \tau^{-1} \alpha^{-1}}$.

Since the circle space has two connected components (namely, $\Delta$ and $g^{-1}(\Sigma \backslash$ $\Delta) f), \gamma$ can either fix both components or interchange these components. In summary, we obtain four possible cases:

(1) $(x, y) \mapsto(\alpha(x), \beta(y))$ is an automorphism that preserves (+)- and (-)parallel classes and fixes each of the two connected components of the circle space if and only if $\beta \Delta \alpha^{-1} \subseteq \Delta$ and $g \beta g^{-1}(\Sigma \backslash \Delta)\left(f \alpha f^{-1}\right)^{-1} \subseteq \Sigma \backslash \Delta$.

(2) $(x, y) \mapsto(\alpha(x), \beta(y))$ is an automorphism that preserves (+)- and (-)parallel classes and exchanges the two connected components of the circle space if and only if $g \beta \Delta(f \alpha)^{-1} \subseteq \Sigma \backslash \Delta$ and $\beta g^{-1}(\Sigma \backslash \Delta)\left(\alpha f^{-1}\right)^{-1} \subseteq \Delta$.

(3) $(x, y) \mapsto(\alpha(y), \beta(x))$ is an automorphism that exchanges (+)- and (-)parallel classes and leaves each connected component of the circle space invariant if and only if $\beta \Delta \alpha^{-1} \subseteq \Delta$ and $g \beta f^{-1}(\Sigma \backslash \Delta)\left(f \alpha g^{-1}\right)^{-1} \subseteq \Sigma \backslash \Delta$. (Note that $\Delta^{-1}=\Delta$ and $(\Sigma \backslash \Delta)^{-1}=\Sigma \backslash \Delta$.)

(4) $(x, y) \mapsto(\alpha(y), \beta(x))$ is an automorphism that exchanges the two connected components of the circle space and also exchanges $(+)$ - and $(-)$-parallel classes if and only if $g \beta \Delta(f \alpha)^{-1} \subseteq \Sigma \backslash \Delta$ and $\beta f^{-1}(\Sigma \backslash \Delta)\left(\alpha g^{-1}\right)^{-1} \subseteq \Delta$.

The following Lemma shows that these conditions severely restrict the possible forms of $\alpha$ and $\beta$.

3.2. Lemma. Let $\Theta=\{x \mapsto x+t \mid t \in \mathbb{R}\} \leq \Delta$. We assume that $\phi \Theta \psi^{-1} \subseteq \Sigma$ for two homeomorphisms $\phi, \psi$ of $\mathbb{S}^{1}$. Then $\phi, \psi \in \Sigma$.

Proof. Replacing $\phi$ and $\psi$ by $\sigma \phi$ and by $\tau \psi$ respectively for suitable $\sigma, \tau \in \Sigma$, we may assume that $\phi$ and $\psi$ both fix $\infty, 1$ and 0 . For each $t \in \mathbb{R}$ there then exist $a_{t}, b_{t}, c_{t}, d_{t} \in \mathbb{R}, a_{t} d_{t}-b_{t} c_{t} \neq 0$, such that

$$
\phi(x+t)=\frac{a_{t} \psi(x)+b_{t}}{c_{t} \psi(x)+d_{t}}
$$

for all $x \in \mathbb{S}^{1}$. Evaluating both sides at $x=\infty$ and $x=0$ gives us $c_{t}=0$ and $\frac{b_{t}}{d_{t}}=\phi(t)$. For $t=0$ we then obtain $\phi(x)=\frac{a_{0}}{d_{0}} \psi(x)$. Evaluating at $x=1$ yields $\frac{a_{0}}{d_{0}}=1$ and thus $\phi=\psi$. Let $\alpha_{t}=\frac{a_{t}}{d_{t}}$. Then

$$
\phi(x+t)=\alpha_{t} \phi(x)+\phi(t)
$$

for all $x, t \in \mathbb{R}$. Since the left-hand side is symmetrical in $x$ and $t$, we find that

$$
\alpha_{t}=\gamma \phi(t)+1
$$


for some constant $\gamma \in \mathbb{R}$. Since $\alpha_{t} \neq 0$ for all $t \in \mathbb{R}$, we must have $\gamma=0$. (Otherwise $t_{0}=\phi^{-1}\left(-\frac{1}{\gamma}\right)$ is defined and $\alpha_{t_{0}}=0$.) Hence $\alpha_{t}=1$ is constant. Therefore the restriction of $\phi$ to $\mathbb{R}$ is additive. So $\phi=\psi=i d$ (note that $\phi(1)=1$ ). In particular, $\phi, \psi \in \Sigma$.

Since $\sigma \Theta$ is a coset of $\Theta$ entirely contained in $\Sigma \backslash \Delta$ for $\sigma \in \Sigma \backslash \Delta$ one readily obtains

\subsection{Corollary.}

(1) $(x, y) \mapsto(\alpha(x), \beta(y))$ is an automorphism that preserves ( + )- and $(-)$ parallel classes and fixes each of the two connected components of the circle space if and only if $\alpha \in \Sigma \cap f^{-1} \Sigma f, \beta \in \Sigma \cap g^{-1} \Sigma g$, and $\beta \alpha^{-1} \in \Delta$ (equivalently, $\beta \alpha^{-1}$ is orientation-preserving).

(2) $(x, y) \mapsto(\alpha(x), \beta(y))$ is an automorphism that preserves ( + )- and ( - parallel classes and exchanges the two connected components of the circle space if and only if $\alpha \in f^{-1} \Sigma \cap \Sigma f, \beta \in g^{-1} \Sigma \cap \Sigma g$, and $\beta \alpha^{-1} \in g^{-1}(\Sigma \backslash \Delta) f$ (equivalently, $\beta \alpha^{-1}$ is orientation-reversing).

(3) $(x, y) \mapsto(\alpha(y), \beta(x))$ is an automorphism that exchanges ( +$)$ - and ( - parallel classes and leaves each connected component of the circle space invariant if and only if $\alpha \in \Sigma \cap f^{-1} \Sigma g, \beta \in \Sigma \cap g^{-1} \Sigma f$, and $\beta \alpha^{-1} \in \Delta$ (equivalently, $\beta \alpha^{-1}$ is orientation-preserving).

(4) $(x, y) \mapsto(\alpha(y), \beta(x))$ is an automorphism that exchanges the two connected components of the circle space and also exchanges $(+)$ - and (-)-parallel classes if and only if $\alpha \in f^{-1} \Sigma \cap \Sigma g, \beta \in g^{-1} \Sigma \cap \Sigma f$, and $\beta \alpha^{-1} \in g^{-1}(\Sigma \backslash \Delta) f$ (equivalently, $\beta \alpha^{-1}$ is orientation-reversing).

3.4. Up to conjugation in $\Sigma$, closed connected subgroups of $\Delta$ are of the following form.

3-dimensional subgroups. Obviously, there is only one such subgroup. This is the group $\Delta$.

2-dimensional subgroups. According to Brouwer's classification of locally compact, connected, transitive and effective transformation groups of $\mathbb{S}^{1}$, cf. [6, Hilfssatz 1.9], a closed connected subgroup 2-dimensional cannot be transitive. So, each such subgroup is contained in the stabilizer of one point. Hence it is conjugate to $L_{2}=\{x \mapsto a x+b \mid a, b \in \mathbb{R}, a>0\}$.

1-dimensional subgroups. Such a subgroup can either act transitively on $\mathbb{S}^{1}$ or it has a fixed point. In the former case the subgroup must be isomorphic to $\mathrm{SO}_{2}(\mathbb{R})$ and so be a maximal compact subgroup of $\Delta$. Furthermore, all maximal compact subgroups are conjugate to each other; cf. [3, Lemma 3.12]. In the latter case the subgroup is isomorphic to $\mathbb{R}$ and is, up to conjugation, a subgroup of $L_{2}$ (with fixed point $\infty$ ). Furthermore, this subgroup then can either act transitively on $\mathbb{R}=\mathbb{S}^{1} \backslash\{\infty\}$ or have a second fixed point. Hence, there are three differnt types of 
closed connected 1-dimensional subgroups.

(1) subgroups transitive on $\mathbb{S}^{1}$ : each such subgroup is conjugate to

$$
\Omega=\left\{x \mapsto \frac{x \cos t-\sin t}{x \sin t+\cos t} \mid t \in \mathbb{R}, 0 \leq t<2 \pi\right\} \simeq \mathrm{SO}_{2}(\mathbb{R}) ;
$$

(2) subgroups fixing precisely one point: each such subgroup is conjugate to

$$
\Theta=\{x \mapsto x+t \mid t \in \mathbb{R}\} \simeq \mathbb{R}
$$

(3) subgroups fixing precisely two points: each such subgroup is conjugate to

$$
\Lambda=\{x \mapsto r x \mid r \in \mathbb{R}, r>0\} \simeq \mathbb{R} .
$$

3.5. Lemma. Let $\Omega=\left\{x \mapsto \frac{x \cos t-\sin t}{x \sin t+\cos t} \mid t \in \mathbb{R}, 0 \leq t<2 \pi\right\} \leq \Delta$ and assume that $\phi \Omega \phi^{-1} \subseteq \Sigma$ for a homeomorphism $\phi$ of $\mathbb{S}^{1}$. Then $\phi \in \Sigma$.

Proof. Let $\rho_{t}$ denote the mapping $x \mapsto \frac{x \cos t-\sin t}{x \sin t+\cos t}$. Since $\phi \Omega \phi^{-1} \simeq \mathrm{SO}_{2}(\mathbb{R})$ is conjugate to $\Omega$ in $\Delta$ and because automorphisms of $\Omega$ can be obtained by conjugation in $\Sigma$, there is a $\sigma \in \Sigma$ such that $\phi \rho_{t}=\sigma \rho_{t} \sigma^{-1} \phi$ for all $t \in \mathbb{R}$. Let $\psi=\sigma^{-1} \phi$. Then $\psi$ commutes with $\Omega$ elementwise, that is, $\psi \rho_{t}=\rho_{t} \psi$ for all $t \in \mathbb{R}$. Evaluating both mappings at $x=0$ gives us $\psi(-\tan t)=\frac{\psi(0)-\tan t}{\psi(0) \tan t+1}$, that is, $\psi=\left(\begin{array}{cc}1 & \psi(0) \\ -\psi(0) & 1\end{array}\right)$ describes a member of $\Sigma$. In particular, $\phi=\sigma \psi \in \Sigma$.

3.6. Lemma. Let $\Lambda=\{x \mapsto r x \mid r \in \mathbb{R}, r>0\} \leq \Delta$ and assume that $\phi \Lambda \phi^{-1} \subseteq \Sigma$ for a homeomorphism $\phi$ of $\mathbb{S}^{1}$. Then $\phi \in \Sigma f_{p, q}$ for some $p, q \in \mathbb{R}^{+}$.

Proof. Without loss of generality we may assume that $\phi$ fixes $\infty, 1$ and 0 . For each $r \in \mathbb{R}^{+}$there exist $a_{r}, b_{r}, c_{r}, d_{r} \in \mathbb{R}, a_{t} d_{t}-b_{t} c_{t} \neq 0$, such that

$$
\phi(r x)=\frac{a_{r} \phi(x)+b_{r}}{c_{r} \phi(x)+d_{r}}
$$

for all $x \in \mathbb{S}^{1}$. Evaluating both sides at $x=\infty, x=0$ and $x=1$ gives us $c_{r}=b_{r}=0$ and $\phi(r)=\frac{a_{r}}{d_{r}}$. Then

$$
\phi(r x)=\phi(r) \phi(x)
$$

for all $x, r \in \mathbb{R}, r>0$. Hence, $\phi=f_{p, q}$ (as defined after Theorem 1.1) for some $p, q \in \mathbb{R}^{+}$.

3.7. The connected component $\Gamma_{f, g}^{1}$ of the identity of $\Gamma_{f, g}$ consists of mappings of the form 3.3.(1). We define the projections $\pi_{1}, \pi_{2}$ by $\pi_{1}(\gamma)=\alpha \in \Sigma \cap f^{-1} \Sigma f$ and $\pi_{2}(\gamma)=\beta \in \Sigma \cap g^{-1} \Sigma g$ where $\gamma$ is described by $\alpha$ and $\beta$ as in 3.3.(1). These are continuous homomorphisms from $\Gamma_{f, g}^{1}$ to $\Sigma$. Since $\pi_{i}\left(\Gamma^{1}\right)$ is connected it follows that $\alpha \in \Delta \cap f^{-1} \Delta f$ and $\beta \in \Delta \cap g^{-1} \Delta g$. So $\pi_{i}$ maps $\Gamma_{f, g}^{1}$ to $\Delta$. Moreover, $\Gamma_{f, g}^{1}$ can be identified with a subgroup of $\Delta \times \Delta$. More precisely, $\Gamma_{f, g}^{1} \cong \pi_{1}\left(\Gamma_{f, g}^{1}\right) \times \pi_{2}\left(\Gamma_{f, g}^{1}\right)$. This proves the following 
Lemma. $(x, y) \mapsto(\alpha(x), \beta(y))$ defines an automorphism of $\mathcal{M}(f, g)$ in the connected component of the identity if and only if $\alpha$ and $\beta$ belong to the connected component of the identity of $\Delta \cap f^{-1} \Delta f$ and $\Delta \cap g^{-1} \Delta g$ respectively. In particular, $\operatorname{dim} \Gamma_{f, g}=\operatorname{dim}\left(\Delta \cap f^{-1} \Delta f\right)+\operatorname{dim}\left(\Delta \cap g^{-1} \Delta g\right)$.

3.8. Corollary. The following are equivalent.

(1) $f$ or $g$ belongs to $\Delta$;

(2) $\mathcal{M}(f, g)$ has a 3-dimensional kernel;

(3) $\Gamma_{f, g}$ is at least 3-dimensional;

(4) at least one of $\Delta \cap f^{-1} \Delta f$ or $\Delta \cap g^{-1} \Delta g$ is 2-dimensional;

(5) $\Delta \cap f^{-1} \Delta f$ or $\Delta \cap g^{-1} \Delta g$ contains a subgroup conjugate to $\Theta$ or to $\Omega$ (see 3.4 for these subgroups).

Proof. If $f$ or $g$ belongs to $\Delta$ then $\mathcal{M}(f, g)$ is a plane with 3-dimensional kernel by Remark 2.7 and Theorem 2.2. Obviously, (2) implies (3).

Suppose that $\operatorname{dim} \Gamma_{f, g}^{1} \geq 3$. Then at least one of $\Delta \cap f^{-1} \Delta f$ or $\Delta \cap g^{-1} \Delta g$ must be of dimension at least 2 by Lemma 3.7. Without loss of generality assume that $\operatorname{dim} \Delta \cap g^{-1} \Delta g \geq 2$. According to the list given in 3.4 either $\Delta \cap g^{-1} \Delta g=\Delta$ or the connected component of the identity of $\Delta \cap g^{-1} \Delta g$ is conjugate to $L_{2}$. In both cases $\Delta \cap g^{-1} \Delta g$ contains a subgroup conjugate to $\Theta$.

Now suppose that $\Delta \cap g^{-1} \Delta g$ contains a subgroup conjugate to $\Theta$ or $\Omega$. Then $g \in \Sigma$ by Lemma 3.2 and Lemma 3.5 respectively. Furthermore, $g \in \Delta$ since $g$ is orientation-preserving.

Let $\mathcal{S}_{0, \infty}$ be the collection of all homeomorphisms of $\mathbb{S}^{1}$ of the form $f_{r, s}$ with $r, s \in \mathbb{R}^{+}$. We then define $\mathcal{S}=\Delta \mathcal{S}_{0, \infty}$. Also let $\mathcal{H}^{+}$be the collection of all orientation-preserving homeomorphisms of $\mathbb{S}^{1}$. With this notation we obtain

3.9. Theorem. The automorphism group of a Minkowski plane $\mathcal{M}(f, g)$ is

- 6-dimensional if and only if $f, g \in \Delta$;

- 4-dimensional if and only if $f \in \Delta, g \in \mathcal{S} \backslash \Delta$ or $g \in \Delta, f \in \mathcal{S} \backslash \Delta$;

- 3-dimensional if and only if $f \in \Delta, g \in \mathcal{H}^{+} \backslash \mathcal{S}$ or $g \in \Delta, f \in \mathcal{H}^{+} \backslash \mathcal{S}$;

- 2-dimensional if and only if $f, g \in \mathcal{S} \backslash \Delta$;

- 1-dimensional if and only if $f \in \mathcal{S} \backslash \Delta, g \in \mathcal{H}^{+} \backslash \mathcal{S}$ or $g \in \mathcal{S} \backslash \Delta, f \in \mathcal{H}^{+} \backslash \mathcal{S}$;

- 0 -dimensional if and only if $f, g \in \mathcal{H}^{+} \backslash \mathcal{S}$.

Proof. By Corollary 3.8 a plane $\mathcal{M}(f, g)$ has an automorphism group of dimension at least 3 if and only if $f$ or $g$ belongs to $\Delta$. In this case $\mathcal{M}(f, g)$ is isomorphic to a plane $\mathcal{M}(h, i d)$ where $h$ is one of the homeomorphisms $f$ or $g$ (the other being in $\Delta)$. Now by Theorem 1.2 and Remark 2.7 the automorphism group of $\mathcal{M}(h, i d)$ is 3-dimensional unless $h$ is of the form $\Delta f_{r, 1}$ for some $r>0$. In the latter case, the automorphism group is 4-dimensional if $r \neq 1$. For $r=1$ one obtains the classical real Minkowski plane, which has a 6-dimensional automorphism group.

Suppose that $\operatorname{dim} \Gamma_{f, g}=2$. Then neither $\Delta \cap f^{-1} \Delta f$ nor $\Delta \cap g^{-1} \Delta g$ can be 2-dimensional and neither contains a subgroup conjugate to $\Theta$ or $\Omega$ by Corollary 3.8. Therefore, both of $\Delta \cap f^{-1} \Delta f$ and $\Delta \cap g^{-1} \Delta g$ must be 1-dimensional and both must be conjugate to $\Lambda$. Since $f$ is orientation-preserving, it follows from Lemma 
3.6 that $f \in \Delta f_{r, s}$ for some $r, s \in \mathbb{R}^{+}$. Similarly, $g \in \Delta f_{r^{\prime}, s^{\prime}}$ for some $r^{\prime}, s^{\prime} \in \mathbb{R}^{+}$. Furthermore, neither $f$ nor $g$ belongs to $\Delta$ by Corollary 3.8.

Suppose that $\operatorname{dim} \Gamma_{f, g}=1$. Then by Lemma 3.7 and Corollary 3.8 we find that one of $\Delta \cap f^{-1} \Delta f$ or $\Delta \cap g^{-1} \Delta g$ must be conjugate to $\Lambda$ and the other must be discrete. Without loss of generality we may assume that $\Delta \cap f^{-1} \Delta f$ is conjugate to $\Lambda$ and that $\Delta \cap g^{-1} \Delta g$ is discrete. Since $f$ is orientation-preserving, it follows from Lemma 3.6 that $f \in \Delta f_{r, s}$ for some $r, s \in \mathbb{R}^{+}$. Moreover $f \notin \Delta$ by Corollary 3.8. By Lemmata $3.2,3.5$ and $3.6 g$ cannot be of the form $\Delta f_{r, s}$.

3.10. All automorphisms of $\mathcal{M}(f, g)$ can be determined from Corollary 3.3. We shall construct a family of Minkowski planes that admit no other automorphisms than the identity. By Theorem 3.9 we necessarily must have $f, g \in \mathcal{H}^{+} \backslash \mathcal{S}$.

Note that $\sigma \in \Sigma \cap g^{-1} \Sigma f$ implies that $\sigma^{-1} \in \Sigma \cap f^{-1} \Sigma g$ and $\sigma^{2} \in \Delta$. Thus $(x, y) \mapsto\left(\sigma^{-1}(y), \sigma(x)\right)$ is an automorphism of $\mathcal{M}(f, g)$ by Corollary 3.3.(3). Hence, excluding automorphisms of this kind, is equivalent to $\Sigma \cap g^{-1} \Sigma f=\emptyset$.

Similarly, excluding automorphisms of the kind described in 3.3.(4) is equivalent to $g^{-1} \Delta \cap \Delta f=\emptyset$ or $g^{-1}(\Sigma \backslash \Delta) \cap(\Sigma \backslash \Delta) f=\emptyset$. If, for example, $g^{-1} \Delta \cap \Delta f=\emptyset$, then also $f^{-1} \Delta \cap \Delta g=\emptyset$. So in order that $(x, y) \mapsto(\alpha(y), \beta(x))$ is an automorphism we necessarily have $\alpha \in f^{-1}(\Sigma \backslash \Delta) \cap(\Sigma \backslash \Delta) g$ and $\beta \in g^{-1}(\Sigma \backslash \Delta) \cap(\Sigma \backslash \Delta) f$ in this case. But then $\beta \alpha^{-1}$ is orientation-preserving violating the condition given in Corollary 3.3.(4).

Considering possible automorphisms of type 3.3.(1) and 3.3.(2) we obtain

Lemma. $\mathcal{M}(f, g)$ admits no other automorphism than the identity if and only if the following holds.

(1) $\Sigma \cap f^{-1} \Sigma f=\Sigma \cap g^{-1} \Sigma g=\{i d\}$;

(2) $f^{-1} \Delta \cap \Delta f=\emptyset, g^{-1}(\Sigma \backslash \Delta) \cap(\Sigma \backslash \Delta) g=\emptyset$ or $f^{-1}(\Sigma \backslash \Delta) \cap(\Sigma \backslash \Delta) f=\emptyset$, $g^{-1} \Delta \cap \Delta g=\emptyset$

(3) $\Sigma \cap g^{-1} \Sigma f=\emptyset$;

(4) $g^{-1} \Delta \cap \Delta f=\emptyset$ or $g^{-1}(\Sigma \backslash \Delta) \cap(\Sigma \backslash \Delta) f=\emptyset$.

We will construct homeomorphisms $f, g$ such that $h \Sigma \cap \Sigma k=\emptyset$ for all distinct $h, k \in\left\{f, f^{-1}, g, g^{-1}\right\}$ and $h \Sigma \cap \Sigma h=\{h\}$ for $h=f, g$. According to the above Lemma this guarantees us that the corresponding 2-dimensional Minkowski plane $\mathcal{M}(f, g)$ has trivial automorphism group.

Let $\mu_{r}$, where $r \neq 0$, be defined by $\mu_{r}(x)=r x$ for all $x \in \mathbb{R}$ and $\mu_{r}(\infty)=\infty$. Also, let $\rho$ and $\eta$ denote the reflections

$$
\rho(x)= \begin{cases}1-x, & \text { if } x \\ \infty, & \text { if } x=\infty\end{cases}
$$

and

$$
\eta(x)= \begin{cases}\frac{1}{x}, & \text { if } x \neq 0, \infty \\ \infty, & \text { if } x=0 \\ 0, & \text { if } x=\infty\end{cases}
$$


$\rho$ and $\eta$ are involutions. Furthermore, $\rho \eta$ has order 3 so that $\rho \eta \rho=\eta \rho \eta$. The group generated by $\rho$ and $\eta$ is isomorphic to the symmetric group on three elements. It consists of the mappings $i d, \rho, \eta, \rho \eta, \eta \rho, \rho \eta \rho$. Furthermore, this group is the subgroup of $\Sigma$ leaving $\{0,1, \infty\}$ invariant.

For $a, b, c>0$ we define the orientation-preserving homeomorphism $h_{a, b, c}$ of $\mathbb{S}^{1}=\mathbb{R} \cup\{\infty\}$ by

$$
h_{a, b, c}(x)=\left\{\begin{array}{ll}
\rho \mu_{a} \rho(x), & \text { if } x \in \mathbb{R}, x \geq 1 \\
\eta \rho \mu_{b} \rho \eta, & \text { if } x \in \mathbb{R}, 0 \leq x \leq 1 \\
\mu_{c}(x), & \text { if } x \in \mathbb{R}, x \leq 0 \\
\infty, & \text { if } x=\infty
\end{array} .\right.
$$

$h_{a, b, c}$ is a piecewise fractional linear mapping that fixes $0,1, \infty$. More precisely, let $A=\{x \in \mathbb{R} \mid x>1\}, B=\{x \in \mathbb{R} \mid 0<x<1\}$ and $C=\{x \in \mathbb{R} \mid x<0\}$. Then the restriction of $h_{a, b, c}$ to the closure of any of $A, B, C$ is induced by a member of $\Delta$. Furthermore, if two parts are induced by the same $\delta \in \Delta$, then $\delta$ must fix each of $0,1, \infty$, that is, $\delta$ is the identity. But then the corresponding parameters $a, b$ or $c$ equal 1.

3.11. Lemma. Let $a, b, c, a^{\prime}, b^{\prime}, c^{\prime}>0$ such that at most one of $a, b, c$ equals 1 . Then $h_{a^{\prime}, b^{\prime}, c^{\prime}} \Sigma \cap \Sigma h_{a, b, c} \neq \emptyset$ if and only if $\left(a^{\prime}, b^{\prime}, c^{\prime}\right)=(a, b, c),\left(b, a, \frac{1}{c}\right),\left(c, \frac{1}{b}, a\right)$, $\left(\frac{1}{c}, \frac{1}{a}, b\right),\left(\frac{1}{b}, c, \frac{1}{a}\right)$ or $\left(\frac{1}{a}, \frac{1}{c}, \frac{1}{b}\right)$. In each of these cases $h_{a^{\prime}, b^{\prime}, c^{\prime}} \Sigma \cap \Sigma h_{a, b, c}$ has precisely one member. In particular, $\left\{a^{\prime}, b^{\prime}, c^{\prime}\right\} \subseteq\left\{a, b, c, \frac{1}{a}, \frac{1}{b}, \frac{1}{c}\right\}$ if $h_{a^{\prime}, b^{\prime}, c^{\prime}} \Sigma \cap \Sigma h_{a, b, c} \neq \emptyset$.

Proof. Suppose there are $\sigma, \tau \in \Sigma$ such that $\tau h_{a, b, c}=h_{a^{\prime}, b^{\prime}, c^{\prime}} \sigma$. Both sides of this identity describe piecewise fractional linear mappings. The fractional linear parts are glued together at $0,1, \infty$ and $\sigma^{-1}(0), \sigma^{-1}(1), \sigma^{-1}(\infty)$ for the left-hand side and right-hand side respectivly. If these points of gluing do not agree, then at least two parts of the mapping on the right-hand side are induced by the same member of $\Sigma$ which implies that at least two of the parameters $a, b, c$ equal 1 - a contradiction to the hypothesis that at most one of $a, b, c$ equals 1 . Hence $\sigma$ leaves $\{0,1, \infty\}$ invariant. Now $\tau=h_{a^{\prime}, b^{\prime}, c^{\prime}} \sigma h_{a, b, c}^{-1}$ maps the elements $0,1, \infty$ like $\sigma$ does. Therefore $\tau=\sigma$. We now have $h_{a^{\prime}, b^{\prime}, c^{\prime}}=\sigma h_{a, b, c} \sigma^{-1}$. Furthermore, $\sigma \in\{i d, \rho, \eta, \rho \eta, \eta \rho, \rho \eta \rho\}$.

One readily obtains that $\rho h_{a, b, c} \rho^{-1}=h_{b, a, \frac{1}{c}}$ and $\eta h_{a, b, c} \eta^{-1}=h_{c, \frac{1}{b}, a}$. From this one finds the remaining entries in the following table where we list $\sigma$ and the parameters $a^{\prime}, b^{\prime}, c^{\prime}$ such that $h_{a^{\prime}, b^{\prime}, c^{\prime}}=\sigma h_{a, b, c} \sigma^{-1}$.

\begin{tabular}{c|cccccc}
$\sigma$ & $i d$ & $\rho$ & $\eta$ & $\rho \eta$ & $\eta \rho$ & $\rho \eta \rho$ \\
\hline$a^{\prime}$ & $a$ & $b$ & $c$ & $\frac{1}{c}$ & $\frac{1}{b}$ & $\frac{1}{a}$ \\
$b^{\prime}$ & $b$ & $a$ & $\frac{1}{b}$ & $\frac{1}{a}$ & $c$ & $\frac{1}{c}$ \\
$c^{\prime}$ & $c$ & $\frac{1}{c}$ & $a$ & $b$ & $\frac{1}{a}$ & $\frac{1}{b}$
\end{tabular}

Conversely, when one of the above six relations between $\left(a^{\prime}, b^{\prime}, c^{\prime}\right)$ and $(a, b, c)$ is satisfied, then it is easy to verify that the corresponding $\sigma$ in the table above satisfies $h_{a^{\prime}, b^{\prime}, c^{\prime}} \sigma=\sigma h_{a, b, c}$. 
3.12. Theorem. Let $a, b, c, a^{\prime}, b^{\prime}, c^{\prime}>0$ such that

(1) $\left\{a, b, c, \frac{1}{a}, \frac{1}{b}, \frac{1}{c}\right\}$ contains at least five distinct elements;

(2) $\left\{a^{\prime}, b^{\prime}, c^{\prime}, \frac{1}{a^{\prime}}, \frac{1}{b^{\prime}}, \frac{1}{c^{\prime}}\right\}$ contains at least five distinct elements;

(3) $\left\{a^{\prime}, b^{\prime}, c^{\prime}\right\} \nsubseteq\left\{a, b, c, \frac{1}{a}, \frac{1}{b}, \frac{1}{c}\right\}$.

Then $\mathcal{M}\left(h_{a, b, c}, h_{a^{\prime}, b^{\prime}, c^{\prime}}\right)$ has no other automorphism than the identity.

Proof. We set $f=h_{a, b, c}$ and $g=h_{a^{\prime}, b^{\prime}, c^{\prime}}$. Then $f^{-1}=h_{\frac{1}{a}, \frac{1}{b}, \frac{1}{c}}$ and $g^{-1}=$ $h_{\frac{1}{a^{\prime}}, \frac{1}{b^{\prime}}, \frac{1}{c^{\prime}}}$. Equating $(a, b, c)$ with $\left(b, a, \frac{1}{c}\right),\left(c, \frac{1}{b}, a\right),\left(\frac{1}{c}, \frac{1}{a}, b\right),\left(\frac{1}{b}, c, \frac{1}{a}\right)$ or $\left(\frac{1}{a}, \frac{1}{c}, \frac{1}{b}\right)$ and $\left(\frac{1}{a}, \frac{1}{b}, \frac{1}{c}\right)$ with $(a, b, c),\left(b, a, \frac{1}{c}\right),\left(c, \frac{1}{b}, a\right),\left(\frac{1}{c}, \frac{1}{a}, b\right),\left(\frac{1}{b}, c, \frac{1}{a}\right)$ or $\left(\frac{1}{a}, \frac{1}{c}, \frac{1}{b}\right)$ yield$\mathrm{s}$ that $\left\{a, b, c, \frac{1}{a}, \frac{1}{b}, \frac{1}{c}\right\}$ can contain at most four distinct elements if one of these identities holds. Therefore hypotheses (1) and (2) imply that $f^{-1} \Sigma \cap \Sigma f=\emptyset$, $\Sigma \cap f^{-1} \Sigma f=f^{-1}(f \Sigma \cap \Sigma f)=\{i d\}$ and $\Sigma \cap g^{-1} \Sigma g=g^{-1}(g \Sigma \cap \Sigma g)=\{i d\}$. Hence the only automorphism of type 3.3.(1) or 3.3.(2) is the identity.

Hypothesis (3) implies that $g^{-1} \Sigma \cap \Sigma f=\emptyset$ and $\Sigma \cap g^{-1} \Sigma f=g^{-1}(g \Sigma \cap \Sigma f)=\emptyset$ by Lemma 3.11. Thus there are no automorphisms of type 3.3.(3) or 3.3.(4). Hence the only automorphism of $\mathcal{M}\left(h_{a, b, c}, h_{a^{\prime}, b^{\prime}, c^{\prime}}\right)$ is the identity.

3.13. Example. Let $b=1, c=a^{2}, a^{\prime}=a, b^{\prime}=1$ and $c^{\prime}=a^{3}$ for $a>0$. Then $a, b, c, a^{\prime}, b^{\prime}, c^{\prime}$ satisfy the hypotheses of Theorem 3.12 for $a \neq 1$. Hence each 2-dimensional Minkowski plane $\mathcal{M}_{a}=\mathcal{M}\left(h_{a, 1, a^{2}}, h_{a, 1, a^{3}}\right), a \neq 1$, admits no other automorphism than the identity. Obviously, one obtains the classical real Minkowski plane for $a=1$.

In the same way as we determined automorphisms of $\mathcal{M}(f, g)$ one can find isomorphisms from $\mathcal{M}\left(f^{\prime}, g^{\prime}\right)$ to $\mathcal{M}(f, g)$. One obtains the following

\subsection{Theorem.}

(1) $(x, y) \mapsto(\alpha(x), \beta(y))$ is an isomorphism from $\mathcal{M}\left(f^{\prime}, g^{\prime}\right)$ to $\mathcal{M}(f, g)$ if and only if

- $\alpha \in \Sigma \cap f^{-1} \Sigma f^{\prime}, \beta \in \Sigma \cap g^{-1} \Sigma g^{\prime}$, and $\beta \alpha^{-1} \in \Delta$, or

- $\alpha \in f^{-1} \Sigma \cap \Sigma f^{\prime}, \beta \in g^{-1} \Sigma \cap \Sigma g^{\prime}$, and $\beta \alpha^{-1} \in g^{-1}(\Sigma \backslash \Delta) f$;

(2) $(x, y) \mapsto(\alpha(y), \beta(x))$ is an isomorphism from $\mathcal{M}\left(f^{\prime}, g^{\prime}\right)$ to $\mathcal{M}(f, g)$ if and only if

- $\alpha \in \Sigma \cap f^{-1} \Sigma g^{\prime}, \beta \in \Sigma \cap g^{-1} \Sigma f^{\prime}$, and $\beta \alpha^{-1} \in \Delta$, or

$-\alpha \in f^{-1} \Sigma \cap \Sigma g^{\prime}, \beta \in g^{-1} \Sigma \cap \Sigma f^{\prime}$, and $\beta \alpha^{-1} \in g^{-1}(\Sigma \backslash \Delta) f$.

Each isomorphism from $\mathcal{M}\left(f^{\prime}, g^{\prime}\right)$ to $\mathcal{M}(f, g)$ is either of the form (1) or (2).

3.15. Example. The 2-dimensional Minkowski planes $\mathcal{M}_{a}$ constructed in 3.13 are pairwise non-isomorphic for $0<a \leq 1 . \mathcal{M}_{1}$ is the classical real Minkowski plane and thus has a non-trivial automorphism group. Therefore $\mathcal{M}_{1}$ cannot be isomorphic to any plane $\mathcal{M}_{a}$ for $0<a<1$. That the other Minkowski planes in this family are pairwise non-isomorphic readily follows from Theorem 3.14 and Lemma 3.11.

3.16. Remark. $\Sigma \cap f^{-1} \Sigma f^{\prime} \neq \emptyset$ implies that $f^{\prime}$ belongs to the double coset $\Sigma f \Sigma$. Checking the other conditions for the existence of an isomorphism from 
$\mathcal{M}\left(f^{\prime}, g^{\prime}\right)$ to $\mathcal{M}(f, g)$ as given in Theorem 3.14 , it follows that $f^{\prime}, g^{\prime}$ both belong to $\Sigma\left\{f, f^{-1}, g, g^{-1}\right\} \Sigma$ if $\mathcal{M}\left(f^{\prime}, g^{\prime}\right)$ is isomorphic to $\mathcal{M}(f, g)$.

\section{REFERENCES}

1. R. Artzy; H. Groh, Laguerre and Minkowski planes produced by dilatations, J. Geom. 6 (1986), $1-20$.

2. E. Hartmann, Beispiele nicht einbettbarer reeller Minkowskiebenen, Geom. Dedicata 10 (1981), 155-159.

3. K. Iwasawa, On some types of topological groups, Ann. of Math. 50 (1949), 507-557.

4. J. Jakóbowski, A full generalization of Moulton's method applied to Minkowski axioms, Zeszyty Nauk. Geom. 20 (1993), 13-21.

5. R. Löwen, Projectivities and the geometric structure of topological planes, in: Geometry - von Staudt's point of view (eds P. Plaumann and K. Strambach), Bad Windsheim (1981), 339-372.

6. H. Salzmann, Zur Klassifikation topologischer Ebenen, Math. Ann. 150 (1963), 226-241.

7. A. Schenkel, Topologische Minkowskiebenen, Dissertation, Erlangen-Nürnberg, 1980.

8. G.F. Steinke, The automorphism group of locally compact connected topological Benz planes, Geom. Dedicata 16 (1984), 351-357.

9. G.F. Steinke, Some Minkowski planes with 3-dimensional automorphism group, J. Geom. 25 (1985), 88-100. 